\title{
Neutrophil Gelatinase-Associated Lipocalin (NGAL) predicts renal injury in acute decompensated cardiac failure: a prospective observational study
}

Stephen Macdonald ${ }^{1,2,3^{*}}$, Glenn Arendts ${ }^{1,2,3}$, Yusuf Nagree ${ }^{3,4}$ and Xiao-Fang $\mathrm{Xu}^{5}$

\begin{abstract}
Background: Acute Decompensated Cardiac Failure (ADCF) is frequently associated with deterioration in renal function. Neutrophil gelatinase-associated lipocalin (NGAL) is an early marker of kidney injury. We aimed to determine if NGAL measured at admission predicts in-hospital acute kidney injury (AKI) in ADCF.

Methods: A prospective observational study measured NGAL and B-natriuretic peptide (BNP) from patients with ADCF presenting to two tertiary hospitals. Patients received standard care and were followed up daily as inpatients. ADCF was defined by PRIDE score $\geq 6$ and AKI by RIFLE criteria.

Results: One hundred and two patients (median age 80, IQR 69-84 years, 52\% male) were enrolled. AKI developed in $22(25 \%)$ of 90 for whom outcome data was available. Seven patients died. NGAL was significantly elevated in those who developed AKI versus those who did not (median $130 \mathrm{ng} / \mathrm{ml}$ vs $69 \mathrm{ng} / \mathrm{ml}, p=0.002$ ). NGAL was also higher in those who died (median $136 \mathrm{ng} / \mathrm{ml}$ vs $68 \mathrm{ng} / \mathrm{ml}, p=0.005$ ). AKI was significantly associated with risk of death $(5 / 22(23 \%)$ vs $1 / 68(1.5 \%), p=0.001)$, but not length of hospital stay. NGAL significantly correlated with admission eGFR but not BNP. For prediction of AKI, NGAL > $89 \mathrm{ng} / \mathrm{ml}$ had sensitivity of $68 \%$ and specificity of $70 \%$ with area under the receiver operator characteristic (ROC) curve of 0.71 (0.58-0.84). After adjustment for baseline renal function, the odds ratio (OR) for AKI was 3.73 (1.26-11.01) if admission NGAL > $89 \mathrm{ng} / \mathrm{ml}$.
\end{abstract}

Conclusions: Elevated NGAL at admission is associated with in-hospital AKI and mortality in patients with ADCF. However, it has only moderate diagnostic accuracy in this setting.

\section{Background}

Cardiac failure is frequently complicated by renal impairment and this is associated with worse outcome [1-3]. Renal insufficiency, defined by estimated glomerular filtration rate $(\mathrm{eGFR})<60 \mathrm{ml} / \mathrm{min} / 1.73 \mathrm{~m}^{2}$ had an overall prevalence of $57 \%$ in an analysis of hospitalised patients with heart failure, and was an independent predictor of mortality [4]. In another study of patients admitted to hospital with Acute Decompensated Cardiac Failure (ADCF), 27\% had subsequent deterioration of renal

\footnotetext{
* Correspondence: stephen.macdonald@health.wa.gov.au

${ }^{1}$ Centre for Clinical Research in Emergency Medicine, Western Australian Institute for Medical Research, Level 5, MRF Building, Rear 50 Murray Street, Perth, WA6000, Australia

Full list of author information is available at the end of the article
}

function which was associated with increased mortality and length of stay [5].

Neutrophil gelatinase-associated lipocalin (NGAL) has been shown to be an early marker of acute kidney injury (AKI) in a number of settings [6-9]. Mechanisms of kidney injury in ADCF are multi-factorial, and in some instances may be exacerbated by therapy such as diuretics [10-12]. Identification of patients at risk of subsequent deterioration in renal function may allow for individualised therapy to mitigate this, for example by careful titration of loop diuretic doses and avoidance of potential nephrotoxins such as intravenous radiographic contrast media.

The purpose of this investigation was to determine whether measurement of NGAL at presentation to the Emergency Department (ED) could predict in hospital

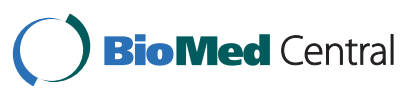


AKI in patients with ADCF, and to assess the utility of this marker in risk-stratification.

\section{Methods \\ Design and setting}

A prospective, observational, un-blinded study conducted in the Emergency Department (ED) of two University hospitals in Western Australia between January 2010 and January 2011.

\section{Population and patient selection}

Patients presenting to the ED with symptoms consistent with ADCF during rostered research nurse hours (7 days per week 0700-2200) were screened for inclusion and underwent testing for BNP to enable calculation of PRIDE acute heart failure score [13]. A modified PRIDE score $\geq 6$ (using a BNP cut-off of $400 \mathrm{pg} / \mathrm{ml}$ rather than NT-proBNP) was considered diagnostic for ADCF. The hospital discharge summary was reviewed to confirm diagnosis. Exclusion criteria were age $<18$ years and end-stage renal failure on dialysis. Informed written consent was obtained from all patients; in patients whose clinical condition at presentation precluded informed discussion, we had provision for 'deferred consent' to enable processing of early blood samples. Ongoing participation in this circumstance required the agreement of the next-of-kin, with consent obtained from the patient as soon as their clinical condition allowed. The study complied with the Declaration of Helsinki and was approved by the Royal Perth Hospital Human Research Ethics Committee (Reference EC 2009/097).

\section{Biomarker analysis}

Blood samples were taken when initial intravenous access was obtained and prior to any intravenous therapy. BNP and NGAL assays were undertaken immediately by trained research personnel, with the Biosite Triage device (Alere, Inverness Medical, Australia Inc) using whole blood. We did not measure urine NGAL as this particular assay is designed only for blood. These results were not made available to clinical staff. Additionally all patients had electrolytes, urea, creatinine and troponin tested on the Abbott Architect (troponin I) or Roche Elecsys (troponin T) laboratory analyser. The NGAL assay used had a lower limit of detection of $60 \mathrm{ng} / \mathrm{ml}$, and a manufacturer quoted $95^{\text {th }}$ percentile of $149 \mathrm{ng} / \mathrm{ml}$, which was used as a dichotomous cut off where appropriate for the purposes of categorical analysis. Baseline estimated glomerular filtration rate (eGFR) was calculated using the admission creatinine level by the Modification of Diet in Renal Disease (MDRD) formula [14].

\section{Inpatient follow up}

Patients were followed up daily by a research nurse. Clinical data including vital signs, weight, fluid balance, and significant investigations or interventions were noted. Episodes of shock, sepsis, myocardial infarction (determined from hospital discharge summary and according to AHA/ESC criteria [15]), administration of intravenous contrast, inotropic or ventilatory support and renal dialysis were recorded. All medications administered and dose changes were recorded. An increase of $50 \%$ or more of baseline loop diuretic dose for at least 24 hours, or the introduction of one of these agents was considered clinically relevant. Potential nephrotoxic agents such as ACE inhibitors were also noted. Renal function was monitored by urine output and daily serum creatinine. Patients were followed for up to 7 days, or until death/hospital discharge, for determination of AKI. Mortality and duration of hospital stay data was obtained from clinical records.

\section{Outcome measures and sample size calculation}

The primary study outcome was AKI developing during the inpatient stay, defined by RIFLE criteria [16], using the admission creatinine as baseline. RIFLE R (Risk) is defined as fall in GFR $\geq 25 \%$ or creatinine rise $\geq 50 \%$ from baseline, or a fall in urine output to $<0.5 \mathrm{ml} / \mathrm{kg} /$ hour for at least 6 hours. Secondary outcome measures were duration of hospital stay and in-hospital mortality. Sample size was determined assuming AKI would develop in $20 \%$ of patients and that elevation of NGAL > $149 \mathrm{ng} / \mathrm{ml}$ (95th percentile) would confer a relative risk of 4 times for this outcome. A total sample of 85 would be sufficient to demonstrate this with power 0.8 and $\alpha 0.05$. We aimed to recruit 100 patients to allow for $15 \%$ margin for missing data etc.

\section{Statistical analysis}

Continuous data is reported as mean \pm SD or median + IQR for parametric and non- parametric distributions respectively and differences analysed by Student's t-test or Mann-Whitney $U$ test as appropriate. Relationships between continuous variables were assessed by Spearman's rank correlation coefficient. For proportions, univariate analyses used Chi squared or Fisher's exact test. Receiver operator characteristic curves were plotted for NGAL, eGFR and creatinine at admission. To adjust for admission eGFR, we used logistic regression analysis. Goodness-of-fit was assessed using the Hosmer and Lemeshow test. All analyses were conducted using SPSS version 17 (SPSS Inc, Chicago, IL, USA). 95\% confidence intervals are quoted and a $\mathrm{p}$ value $<0.05$ considered significant.

\section{Results}

One hundred and two patients were recruited into the study. Median age was 80 years (IQR 69-84 years) and $52(51 \%)$ were male. Baseline characteristics of the study population are shown in Table 1. Twelve patients did 
Table 1 Participant characteristics and past medical history

\begin{tabular}{cc}
\hline $\mathrm{n}$ & 102 \\
\hline Median Age (IQR) & $80(69-84)$ \\
\hline Male & $52(51 \%)$ \\
\hline Median BNP & $723 \mathrm{pg} / \mathrm{ml}(\mathrm{IQR} 337-1235 \mathrm{pg} / \mathrm{ml})$ \\
\hline Median PRIDE Score & $12($ Range 6-14) \\
\hline Congestive Cardiac Failure & $50(49 \%)$ \\
\hline Hypertension & $69(68 \%)$ \\
\hline Coronary Heart Disease & $46(45 \%)$ \\
\hline Valvular Heart Disease & $60(59 \%)$ \\
\hline Chronic Obstructive Airways Disease & $19(19 \%)$ \\
\hline Chronic Renal Impairment (admission eGFR $\left.<60 \mathrm{ml} / \mathrm{min} / 1.73 \mathrm{~m}^{2}\right)$ & $22(22 \%)$ \\
\hline
\end{tabular}

not have subsequent data to enable determination of AKI. These patients were either transferred to another hospital after enrolment in the study, or were admitted but did not have subsequent creatinine measurement prior to discharge or death. Of the remaining 90 patients, 22 (25\%) had in-hospital AKI including one patient who required short-term dialysis in the intensive care unit in the context of cardiogenic shock complicating myocardial infarction. On examination of the hospital discharge diagnosis, ADCF was subsequently not considered to be the primary diagnosis in 4 patients. None of these developed AKI. The flow of participants through the study is summarized in Figure 1.

\section{Relationship between NGAL, eGFR and BNP}

There was a significant relationship between NGAL and admission eGFR (Spearman's rho -0.42, $p=0.01$ ) but not between NGAL and BNP level (Spearman's rho +0.13, $p=$ $0.18)$.

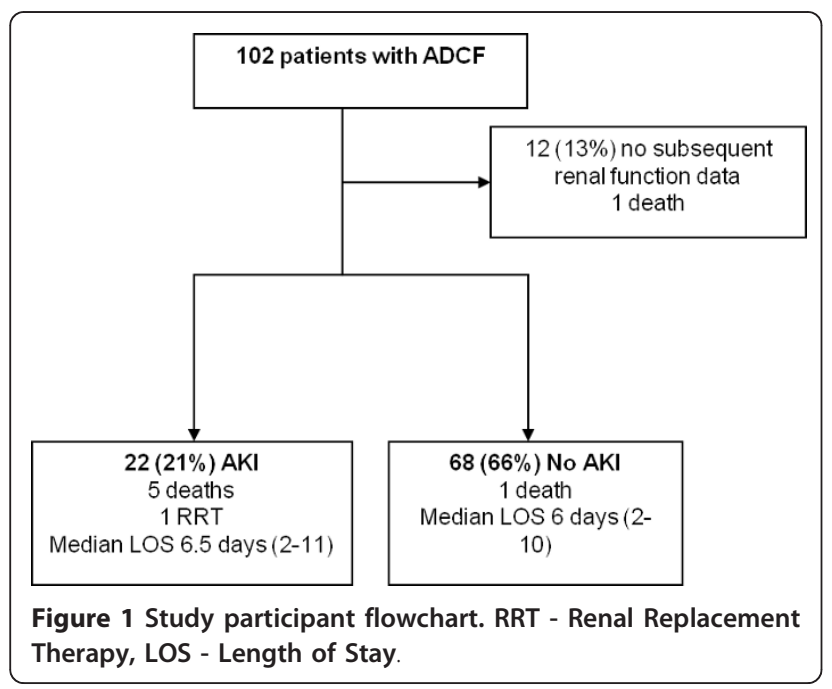

\section{NGAL and AKI}

Figure 2 compares admission NGAL level between those patients who developed in hospital AKI and those who did not. Median NGAL level was $130 \mathrm{ng} / \mathrm{ml}$ (IQR 71-193 ng/ml) for those who developed AKI compared to $69 \mathrm{ng} / \mathrm{ml}$ (IQR $60-103 \mathrm{ng} / \mathrm{ml}$ ) for those who did not $(p=0.002)$.

\section{Mortality}

Seven participants died during the hospital stay. Of these, five had AKI and one did not $(p=0.002)$. One further patient did not have subsequent renal function data to enable classification. Median NGAL was 136 $\mathrm{ng} / \mathrm{ml}$ (IQR $133-170 \mathrm{ng} / \mathrm{ml}$ ) for patients who died versus $68 \mathrm{ng} / \mathrm{ml}$ (IQR $60-119 \mathrm{ng} / \mathrm{ml}$ ) for those who did not $(p=0.005)$.

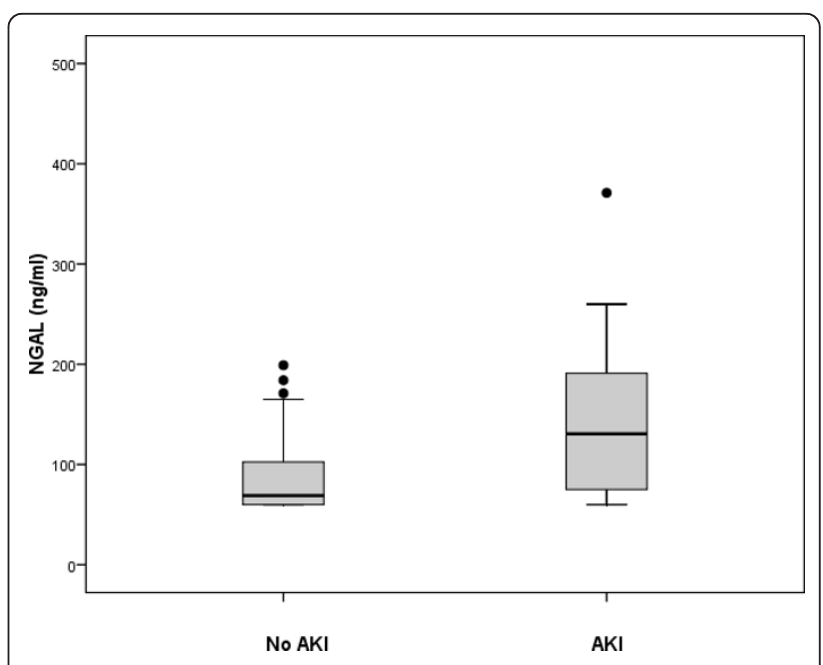

Figure 2 Neutrophil gelatinase associated lipocalin (NGAL) and Acute Kidney Injury. Median NGAL 130 ng/ml (IQR 71-193 ng/ml) in patients who subsequently developed AKI $(n=22)$ versus $69 \mathrm{ng} /$ $\mathrm{ml}($ IQR $60-103 \mathrm{ng} / \mathrm{ml})$ for those who did not $(n=68), p=0.002$ 


\section{Length of hospital stay}

Length of hospital stay was similar between those who developed AKI and those who did not. Median admission duration was 6.5 days (IQR 2-11 days) for the AKI group and 6 days (IQR 2-10 days) for those without AKI ( $p=$ $0.96)$. This did not significantly change when patients who died were excluded.

\section{Predictive value of NGAL for AKI}

Table 2 shows results of univariate analysis of variables considered, a priori, likely to be associated with risk of developing AKI. The manufacturer quoted cut off at the 95th percentile for NGAL is $149 \mathrm{ng} / \mathrm{ml}$. In the present study this value corresponded to a sensitivity of $45 \%$ and specificity of $85 \%$ for AKI. A receiver operator characteristic (ROC) curve (Figure 3) showed an optimal NGAL cut off at $>89 \mathrm{ng} / \mathrm{ml}$, with sensitivity of $68 \%$ and specificity of $70 \%$ for AKI, with area under the curve (AUC) of 0.71 (0.58-0.84). The AUC of the ROC curve for admission eGFR was $0.72(0.61-0.83)$ and for admission creatinine $0.70(0.58-0.82)$. In addition to NGAL, baseline renal impairment (as defined by admission eGFR $<60 \mathrm{ml} / \mathrm{min} / 1.73 \mathrm{~m}^{2}$ ) was significantly associated with AKI (Table 2). Four patients were subsequently considered not to have ADCF. All of these had NGAL < $89 \mathrm{ng} / \mathrm{ml}$ and none developed ADCF. Excluding these from the analysis did not significantly alter the results. After adjustment for admission eGFR, odds ratio (OR) for AKI was 3.73 (1.26-11.01) for NGAL > $89 \mathrm{ng} / \mathrm{ml}$, and $4.78(1.49-15.30)$ for NGAL > $149 \mathrm{ng} / \mathrm{ml}$. NGAL was $>89 \mathrm{ng} / \mathrm{ml}$ in 14 of 19 patients $(74 \%)$ with admission eGFR $<60 \mathrm{ml} / \mathrm{min} / 1.73 \mathrm{~m}^{2}$ who developed AKI, and in one of 3 (33\%) of those with admission eGFR $\geq$ $60 \mathrm{ml} / \mathrm{min} / 1.73 \mathrm{~m}^{2}$ who developed AKI.

\section{Discussion}

The present study confirms that AKI is a common complication of ADCF. Median NGAL is significantly higher at admission in patients who developed AKI and in those who died. Elevated NGAL at admission is associated with AKI after adjustment for eGFR. AKI is associated with increased risk of death but we did not find any association with increased length of hospital stay as has been found by other investigators [5]. This may have been a chance finding as the study was not powered to assess these outcomes. Although NGAL identifies a higher risk subgroup of patients for development of AKI, in this study it was found to have only moderate diagnostic accuracy.

Aghel et al [17] obtained NGAL levels on 91 admitted patients with physician adjudicated ADCF. Thirty eight percent of these developed worsening renal function (WRF), defined by a rise in creatinine $>0.3 \mathrm{mg} / \mathrm{dl}$, with NGAL > $140 \mathrm{ng} / \mathrm{ml}$ having an odds ratio of 7.4 for WRF, after adjustment for baseline eGFR. Higher NGAL levels also increased risk of death. This study found admission NGAL to have a sensitivity of $85 \%$ and specificity of $54 \%$, compared to $68 \%$ and $70 \%$ in our study, and found a higher NGAL cut off value. There were important differences from our study which may explain these discrepancies. We enrolled patients on arrival in the ED using a pragmatic diagnostic tool rather than selected by physicians after admission, when diagnostic workup may be more complete. The incidence of renal

Table 2 Univariate analysis of risk factors for AKI

\begin{tabular}{|c|c|c|c|c|}
\hline FACTOR & AKI & No AKI & $\begin{array}{l}\text { Odds ratio } \\
(95 \% \mathrm{Cl})\end{array}$ & $P$ value \\
\hline $\mathrm{NGAL}>89 \mathrm{ng} / \mathrm{ml}$ & $15 / 22(68 \%)$ & $20 / 68(29 \%)$ & $5.1(1.8-14.5)$ & $0.003^{*}$ \\
\hline $\mathrm{NGAL}>149 \mathrm{ng} / \mathrm{ml}$ & $10 / 22(45 \%)$ & $8 / 68(12 \%)$ & $6.1(2.0-18.8)$ & $0.002^{*}$ \\
\hline Age $\geq 80$ years & $10 / 22(45 \%)$ & $33 / 68(49 \%)$ & $1.3(0.4-3.3)$ & 0.81 \\
\hline $\mathrm{eGFR}<60 \mathrm{ml} / \mathrm{min} / 1.73 \mathrm{~m}^{2}$ & $19 / 22(86 \%)$ & $35 / 68(51 \%)$ & $6.0(1.6-22.0)$ & $0.005^{*}$ \\
\hline Diabetes & $10 / 22(45 \%)$ & $32 / 68(47 \%)$ & $0.9(0.4-2.5)$ & 0.90 \\
\hline Hypertension & $16 / 22(72 \%)$ & $45 / 68(66 \%)$ & $1.4(0.5-3.9)$ & 0.57 \\
\hline Loop diuretic $\uparrow^{1}$ & $15 / 22(68 \%)$ & $43 / 68(63 \%)$ & $1.2(0.4-3.5)$ & 0.64 \\
\hline Nephrotoxic drug ${ }^{2}$ & $7 / 22(32 \%)$ & $25 / 68(37 \%)$ & $0.8(0.3-2.2)$ & 0.64 \\
\hline IV Contrast & $6 / 22(27 \%)$ & $15 / 68(22 \%)$ & $1.3(0.4-4.0)$ & 0.64 \\
\hline Sepsis & $1 / 22(4 \%)$ & $1 / 68(1.5 \%)$ & $3.2(0.2-54.0)$ & 0.43 \\
\hline Shock & $2 / 22(9 \%)$ & $4 / 68(6 \%)$ & $1.6(0.3-9.4)$ & 0.63 \\
\hline Myocardial Infarction & $6 / 22(27 \%)$ & $9 / 68(13 \%)$ & $2.5(0.8-8.0)$ & 0.19 \\
\hline Urinary Infection & $3 / 22(14 \%)$ & $5 / 68(7 \%)$ & $2.0(0.4-9.1)$ & 0.40 \\
\hline \multicolumn{5}{|l|}{${ }^{*} p<0.05$} \\
\hline \multicolumn{5}{|c|}{1 Introduction of loop diuretic or increase in usual dose of $\geq 50 \%$ over baseline for at least 24 hours (including bolus doses) } \\
\hline
\end{tabular}




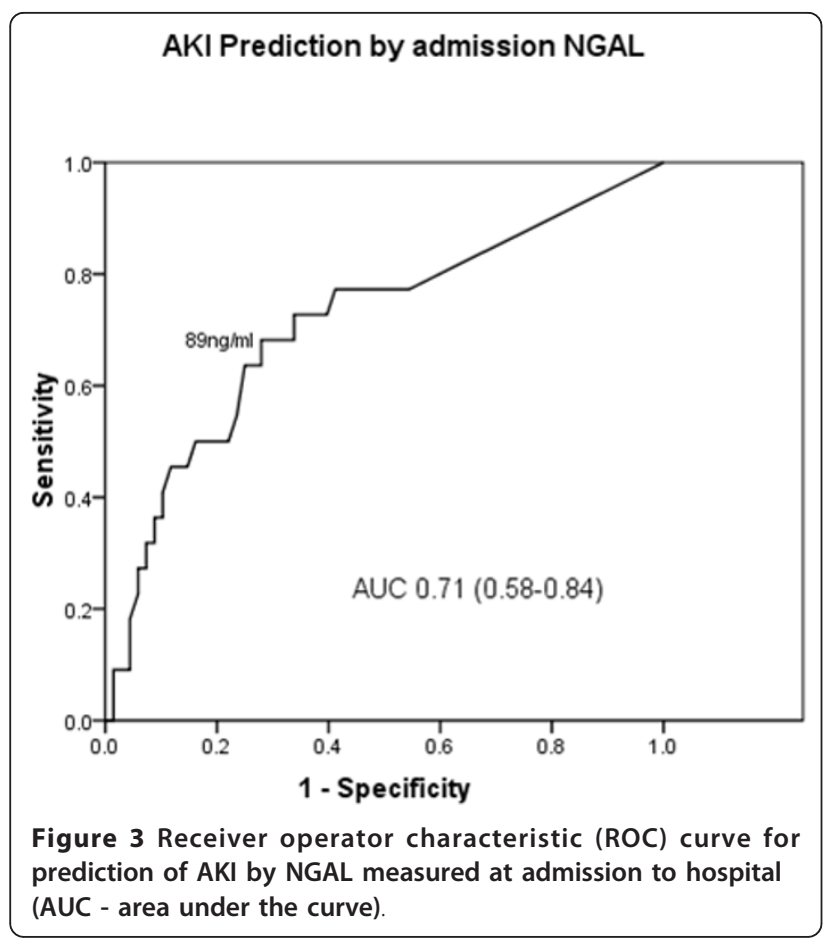

injury was lower in our study (25\% vs 38\%) suggesting possible differences in illness severity, but there was a high prevalence of renal impairment at admission, possibly reflecting the higher median age in our study of 80 years. Finally the assays used were not the same. Differences in calibration of assays and antibodies used may result in markedly different NGAL cut off values.

Unlike other conditions such as post surgery or intravenous contrast administration where NGAL has be shown to be useful for predicting AKI, the time of onset of the renal insult is not easily determined in ADCF. Sixty percent of patients in our study had admission eGFR $<60$ $\mathrm{ml} / \mathrm{min} / 1.73 \mathrm{~m}^{2}$. In the ED setting it is not clear whether this represents chronic impairment or acute injury unless information on stable baseline renal function is available. In some patients, low GFR at admission may represent AKI at its nadir, with subsequent recovery. Eight patients with NGAL > $149 \mathrm{ng} / \mathrm{ml}$ did not develop AKI as defined in this study. Some of these may have had renal injury when compared to their stable baseline eGFR rather than that recorded on admission. In addition, chronic renal impairment itself is a major risk factor for AKI and it is not surprising that low eGFR was significantly associated with this outcome. This finding alone, in the absence of elevated NGAL, should alert the clinician to a high risk of subsequent worsening renal function.

The mechanisms of renal injury in cardiac failure, also termed cardio-renal syndrome, are complex [10-12]. Renal hypoperfusion due to reduced cardiac output or increased venous or abdominal pressures may contribute.
In addition high dose diuretics, intravenous contrast and other drugs may result in renal injury. These factors may explain why some patients who developed AKI in our study did not have elevated NGAL on presentation. NGAL has been shown to be elevated in patients with stable chronic heart failure [18]. While known to be released by renal tubular cells, expression of NGAL by cardiac myocytes has also been demonstrated in heart failure [19]. However a recent clinical study by Shrestha et al [20] found no association between NGAL level and echocardiographic parameters of systolic dysfunction.

\section{Limitations}

Limitations of this study include its un-blinded and observational nature. Data to assess subsequent renal function was not available for all patients. This was most commonly due to no subsequent bloods being taken due to early discharge from hospital or transfer to another facility after recruitment in the ED. In addition, we were restricted in our ability to recruit overnight, a time when acute heart failure presentations are common. The diagnosis of ADCF was based on the clinical judgement of the treating clinician and the PRIDE score rather than formal echocardiography. We used BNP rather than NT-BNP to calculate this score. A BNP $>400 \mathrm{pg} / \mathrm{ml}$ cut point was chosen based on manufacturer recommendation however its incorporation into the PRIDE score has not been validated. This was a pragmatic study reflecting real life clinical practice where initial treatment of ADCF is undertaken in the ED. We did not have information on baseline renal function and used worsening from admission to define AKI with a single NGAL assay. However, ADCF is a dynamic condition and patients present to hospital at varying stages of the illness; obtaining serial NGAL levels, and knowledge of stable baseline renal function prior to acute decompensation would allow further exploration the timing of NGAL peak in relation to nadir of renal function.

\section{Potential clinical implications}

Potential benefits of being able to identify patients at risk of worsening renal function in ADCF are the ability to modify treatment regimes to mitigate this risk. However, a single NGAL level at admission is only moderately accurate, although high levels are associated with higher risk of AKI. The incremental value of NGAL in addition to eGFR estimation in identifying high-risk patients is uncertain. A statistical association with an outcome does not necessarily translate into clinical utility for a biomarker when added to existing clinical and laboratory risk factors [21]. Further studies are required to determine whether knowledge of NGAL level at admission translates into improved outcomes. NGAL may be a tool to unravel the underlying pathophysiology of the cardiorenal syndrome. 


\section{Conclusions}

Elevated NGAL at admission is associated with in-hospital AKI, independent of baseline renal function, in patients admitted from the ED with ADCF and is associated with increased mortality. However, it has only moderate diagnostic accuracy in this setting.

\section{Acknowledgements}

The authors thank Ellen Macdonald and the Emergency Research staff at the participating hospitals. Alere (Inverness Medical Innovations Australia Pty) provided materials and loan of analytical devices and consumables to undertake the BNP and NGAL assays. The company had no role in the study initiation, design, conduct, analysis of data, or decision to publish. The authors had full access to all the data. The study was otherwise supported by existing research resources at the participating institutions

\section{Author details}

${ }^{1}$ Centre for Clinical Research in Emergency Medicine, Western Australian Institute for Medical Research, Level 5, MRF Building, Rear 50 Murray Street, Perth, WA6000, Australia. 'Emergency Medicine, Royal Perth Hospital, GPO Box X2213, Perth, WA6000, Australia. ${ }^{3}$ Emergency Medicine, University of Western Australia, 35 Stirling Highway, Crawley, WA6009, Australia. ${ }^{4}$ Emergency Medicine, Fremantle Hospital, PO Box 480, Fremantle, WA6959, Australia. ${ }^{5}$ Cardiology, Royal Perth Hospital, GPO Box X2213, Perth, WA 6000 Australia.

\section{Authors' contributions}

SM had the original study idea and designed the study with GA and X-FX. All authors undertook data collection and oversaw enrolments. SM and GA undertook the analysis. SM drafted the paper and all authors contributed to its revision. All authors read and approved the final manuscript.

\section{Authors' information}

SM: Emergency Physician and Associate Professor; GA: Emergency Physician and Associate Professor; YN: Emergency Physician and Professor; X-FX Cardiologist.

\section{Competing interests}

SPJM and YN have both spoken at meetings sponsored by Alere (Inverness Medical Innovations Australia Pty). No honoraria or travel costs were claimed. GA and X-FX declare no competing interests. All authors are employees of the Health Department of Western Australia and/or University of Western Australia.

Received: 29 September 2011 Accepted: 17 February 2012

Published: 17 February 2012

\section{References}

1. Adams KF Jr, Fonarow GC, Emerman $\mathrm{CL}$, LeJemtel $\mathrm{TH}$, Costanzo MR, Abraham WT, Berkowitz RL, Galvao M, Horton DP: Characteristics and outcomes of patients hospitalized for heart failure in the United States: rationale, design, and preliminary observations from the first 100,000 cases in the Acute Decompensated Heart Failure National Registry (ADHERE). Am Heart J 2005, 149(2):209-216.

2. Hillege $H L$, Nitsch D, Pfeffer MA, Swedberg K, McMurray JJ, Yusuf S, Granger CB, Michelson EL, Ostergren J, Cornel JH, et al: Renal function as a predictor of outcome in a broad spectrum of patients with heart failure. Circulation 2006, 113(5):671-678.

3. Smith GL, Lichtman JH, Bracken MB, Shlipak MG, Phillips CO, DiCapua P, Krumholz HM: Renal impairment and outcomes in heart failure: systematic review and meta-analysis. J Am Coll Cardiol 2006 47(10):1987-1996.

4. Amsalem Y, Garty M, Schwartz R, Sandach A, Behar S, Caspi A, Gottlieb S, Ezra D, Lewis BS, Leor J: Prevalence and significance of unrecognized renal insufficiency in patients with heart failure. Eur Heart J 2008, 29(8):1029-1036

5. Forman DE, Butler J, Wang Y, Abraham WT, O'Connor CM, Gottlieb SS, Loh E, Massie BM, Rich MW, Stevenson LW, et al: Incidence, predictors at admission, and impact of worsening renal function among patients hospitalized with heart failure. J Am Coll Cardiol 2004, 43(1):61-67.

6. Mishra J, Dent C, Tarabishi R, Mitsnefes MM, Ma Q, Kelly C, Ruff SM, Zahedi K, Shao M, Bean J, et al: Neutrophil gelatinase-associated lipocalin (NGAL) as a biomarker for acute renal injury after cardiac surgery. Lancet 2005, 365(9466):1231-1238

7. Constantin JM, Futier E, Perbet S, Roszyk L, Lautrette A, Gillart T, Guerin R, Jabaudon M, Souweine B, Bazin JE, et al: Plasma neutrophil gelatinaseassociated lipocalin is an early marker of acute kidney injury in adult critically ill patients: a prospective study. J Crit Care 2010, 25(1):176, e1-6.

8. Haase-Fielitz A, Bellomo R, Devarajan P, Story D, Matalanis G, Dragun D, Haase M: Novel and conventional serum biomarkers predicting acute kidney injury in adult cardiac surgery-a prospective cohort study. Critical care medicine 2009, 37(2):553-560.

9. Tuladhar SM, Puntmann VO, Soni M, Punjabi PP, Bogle RG: Rapid detection of acute kidney injury by plasma and urinary neutrophil gelatinaseassociated lipocalin after cardiopulmonary bypass. J Cardiovasc Pharmacol 2009, 53(3):261-266.

10. Sarraf M, Masoumi A, Schrier RW: Cardiorenal syndrome in acute decompensated heart failure. Clin J Am Soc Nephrol 2009, 4(12):2013-2026.

11. Tang WH, Mullens W: Cardiorenal syndrome in decompensated heart failure. Heart 2009, 96(4):255-260

12. Ronco C, Haapio M, House AA, Anavekar N, Bellomo R: Cardiorenal syndrome. J Am Coll Cardiol 2008, 52(19):1527-1539.

13. Baggish AL, Siebert U, Lainchbury JG, Cameron R, Anwaruddin S, Chen A Krauser DG, Tung R, Brown DF, Richards AM, et al: A validated clinical and biochemical score for the diagnosis of acute heart failure: the ProBNP Investigation of Dyspnea in the Emergency Department (PRIDE) acute heart failure score. Am Heart J 2006, 151(1):48-54.

14. Levey AS, Coresh J, Greene T, Stevens LA, Zhang YL, Hendriksen S, Kusek JW, Van Lente F: Using standardized serum creatinine values in the modification of diet in renal disease study equation for estimating glomerular filtration rate. Ann Intern Med 2006, 145(4):247-254

15. Thygesen $\mathrm{K}$, Alpert JS, White HD: Universal definition of myocardial infarction. Eur Heart J 2007, 28(20):2525-2538.

16. Bellomo R, Ronco C, Kellum JA, Mehta RL, Palevsky P: Acute renal failure definition, outcome measures, animal models, fluid therapy and information technology needs: the Second International Consensus Conference of the Acute Dialysis Quality Initiative (ADQI) Group. Crit Care 2004, 8(4):R204-212.

17. Aghel A, Shrestha K, Mullens W, Borowski A, Tang WH: Serum neutrophil gelatinase- associated lipocalin (NGAL) in predicting worsening renal function in acute decompensated heart failure. J Card Fail 2010, 16(1):49-54

18. Damman $K$, van Veldhuisen DJ, Navis G, Voors AA, Hillege HL: Urinary neutrophil gelatinase associated lipocalin (NGAL), a marker of tubular damage, is increased in patients with chronic heart failure. Eur J Heart Fail 2008, 10(10):997-1000

19. Yndestad A, Landro L, Ueland T, Dahl CP, Flo TH, Vinge LE, Espevik T, Froland SS, Husberg C, Christensen G, et al: Increased systemic and myocardial expression of neutrophil gelatinase-associated lipocalin in clinical and experimental heart failure. Eur Heart J 2009, 30(10):1229-1236.

20. Shrestha K, Borowski AG, Troughton RW, Thomas JD, Klein AL, Tang WH: Renal dysfunction is a stronger determinant of systemic neutrophil gelatinase-associated lipocalin levels than myocardial dysfunction in systolic heart failure. J Card Fail 2011, 17(6):472-478.

21. Pencina MJ, D'Agostino RB Sr, D'Agostino RB Jr, Vasan RS: Evaluating the added predictive ability of a new marker: from area under the ROC curve to reclassification and beyond. Statistics in medicine 2008 27(2):157-172, discussion 207-112.

\section{Pre-publication history}

The pre-publication history for this paper can be accessed here: http://www.biomedcentral.com/1471-2261/12/8/prepub

doi:10.1186/1471-2261-12-8

Cite this article as: Macdonald et al:: Neutrophil Gelatinase-Associated Lipocalin (NGAL) predicts renal injury in acute decompensated cardiac failure: a prospective observational study. BMC Cardiovascular Disorders 2012 12:8. 